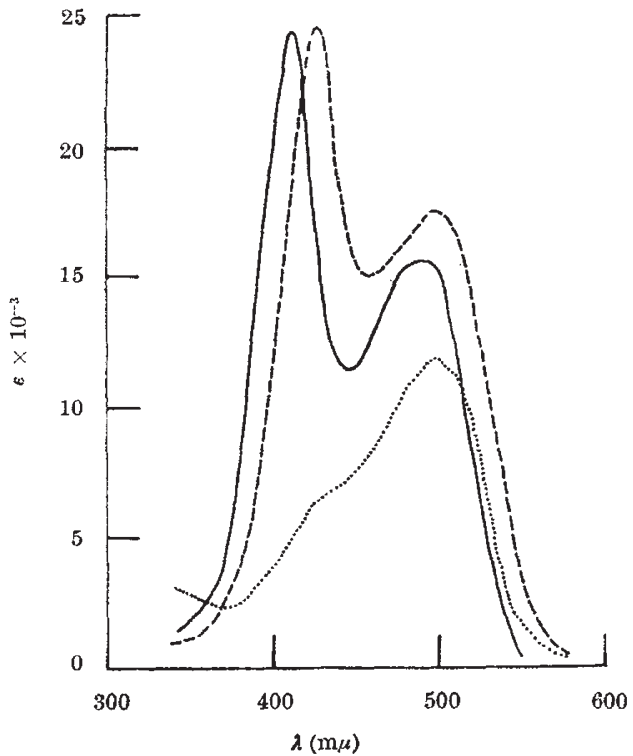

Fig. 1. - Potassium ethyl 1-methoxypicrate $\left(2.27 \times 10^{-5} M\right)$ and potassium ethoxide $\left(6.8 \times 10^{-2} M\right) ;---, 1,3,5$-trinitrobenzene $\left(3 \cdot 23 \times 10^{-5} M\right)$ and potassium ethoxide $\left(6 \cdot 8 \times 10^{-2} M\right.$ within $30 \mathrm{~min}$. of mixing ; ...., the same after $24 \mathrm{hr}$. All solutions

nitrobenzene +ethoxide initially gives an addition product (I, $R=\mathrm{H}$ ) which corresponds with tho Meisenheimer compound.

The spectra were measured on an Optica CF4 grating spectrophotometer.

This work was carried out during the tenure of an Edward A. Deeds Fellowship of the University of St. Andrews.

Chemistry Department,

R. Foster

Que en's College, Dundee.

${ }^{1}$ Ainscough, J. B., and Caldin, E. F., J. Chem. Soc., 2528 (1956) ${ }^{2}$ Meisenheimer, J., Ann. der Chemie, 323, 205 (1902).

'Foster, R., Nature, 176, 746 (1955).

\section{Relation between the Polarizability of Ions and other Significant Properties}

IN $\mathfrak{a}$ previous communication ${ }^{1}$ it was reported that the polarizability $\alpha$ of an ion is proportional to $N^{2}$ ( $N$ being the atomic number of the element). In order to examine the extent to which this relation, $\alpha=k N^{2}$, where $k$ is a constant, is applicable in the case of ions, the data for the polarizabilities of ions ${ }^{2}$ were plotted against the values of $N^{2}$ for the corre sponding ions. It was found that it is applicable for elements in the same sub-group of the periodic table, although the proportionality constant $k$ in this relation for earlier members of each sub-group differs from that applicable to the higher members of the same sub-group.

By applying this criterion, the element fluorine is found to differ from the other halogens, chlorine, bromine and iodine, in having a lower polarizability than that to be expected from the above formula. Similarly, lithium and sodium ions differ from potassium, rubidium and cæsium ions in group $1 A$, beryllium and magnesium differ from calcium, strontium, and barium ions, and so on. Such differences in the behaviour of the earlier elements in the sub-groups from those occurring higher up are in agreement with the corresponding differences in the chemical behaviour between fluorine on one hand and chlorine, bromine and iodine on the other, lithium and sodium from potassium, rubidium and cæsium and so on. This is evidently to be attributed to the electronic configurations in the atoms.

It is found also that for elements belonging to the same series in the different periods, the values of $\alpha$ decrease with increase in $N^{2}$.

Another interesting correlation has been obtained by plotting the values of the electric polarizabilities of ions against their magnetic susceptibilities, $\chi$ (seo ref. 2). The graph thus obtained shows distinct proportionality between the two, the relation being $\alpha=K_{0} \chi$, where $K_{0}$, the constant of proportionality, is different for positive and negative ions.

\section{K. Krishnamurti}

Physico-Chemical Laboratories, College of Science,

University of Baghdad.

${ }^{1}$ Krishnamurti, K., Nature, 182, 1227 (1958).

${ }^{2}$ Data taken mostly from Van Vleck's "Electric and Magnetic Susceptibilities", 225 (Oxf. Univ. Press).

\section{PHYSICS}

\section{Nuclear Magnetic Resonance in Rolled Copper Sheet}

AN investigation has been made of the copper-63 nuclear magnetic resonance in rolled copper sheet. The specimens were made from an electrolytic copper of commercial purity and consisted of a pile of 18 sheets each measuring $0.6 \mathrm{~cm} . \times 0.5 \mathrm{~cm} . \times 0.0045 \mathrm{~cm}$. and insulated from each other by rice paper of thickness $0.003 \mathrm{~cm}$. The measurements were made with a Colpitts marginal oscillator at $a_{0}$ frequency of 5.5 Mc./s., using a permanent magnet with pole pieces of $4 \cdot 5 \mathrm{in}$. diameter and $1 \cdot 5 \mathrm{in}$. gap.

The following features were observed.

(1) In sheet which has been recrystallized after heavy deformation by rolling (90 per cent reduction in thickness or more) the width of the line varies with the orientation of the specimen relative to the main magnetic field. This effect occurs for all annealing temperatures investigated $\left(240-600^{\circ} \mathrm{C}\right.$.) but is more pronounced for the higher temperatures. If the specimen is rotated about the transverse direction (the direction in the plane of the sheet perpendiculax to the rolling direction) the line-width shows minima for $\theta=0$ and $\theta=\pi / 2$, and a maximum for $\theta=\pi / 4$, where $\theta$ is the angle between the main magnetic field and the plane of the sheet. The magnitude of the variation may be as much as \pm 15 per cent in the mean square line-width.

This effect can be explained by the Van Vleck theory of dipolar broadening ${ }^{1}$ if it is assumed that there is present in the specimen a preferred orientation of the type usually observed in recrystallized rolled sheet ${ }^{2}$.

A variation of line-width with $\theta$ is also found for the deformed sheet; this is of a different nature from that in the annealed sheet, and is more difficult to interpret in terms of the expected preferred orienta. tions.

(2) Annealing of a deformed specimen produces no detectable change in the shape of the resonance line, nor in its width except in the case of the most heavily deformed specimens (90 per cent reduction in thick. 